\title{
Fluid Dynamics of Establishing Left-Right Patterning in Development
}

\author{
Julyan H. E. Cartwright, * Nicolas Piro, Oreste Piro and Idan Tuval
}

How does the clockwise motion of tens of monocilia drive a leftward flow in the node? And, as the observed flow is leftward, how is the fluid recirculating within the node, as it must, because the node is a closed structure? How does the nodal flow lead to left-right symmetry breaking in the embryo? These questions are within the realm of fluid physics, whose application to the problem of left-right symmetry breaking in vertebrates has led to important advances in the field. Birth Defects Research (Part C) 84:95-101, 2008. 2008 Wiley-Liss, Inc.

\section{INTRODUCTION}

Unravelling the mysteries of the establishment of left-right patterning in development is an ineluctably interdisciplinary undertaking that encompasses disciplines ranging from molecular biology to fluid dynamics. Particularly interesting and surprising is the now well-accepted fundamental role that fluid dynamical phenomena play at the origin of the chiral differentiation process in some, if not all, vertebrates. In a series of beautiful experiments published from 1998 to 2002, Nonaka et al. (1998, 2002), convincingly showed that the correct lateralization in mice is strongly correlated to the direction of the fluid flow induced within the node by a relatively small number of rotating $9+0$ monocilia attached to the floor of this chamber. In normally developing embryos, this flow proceeds in a well-defined right-left direction, but if it is exogenously forced to reverse, the embryo develops situs inversus (Nonaka et al., 2005). The researchers, however, at that time, could not resolve the conundrum of how a left-right symmetric geometrical configuration of cilia could produce an asymmetric flow.

\section{A MINIMAL MODEL OF NODAL FLOW}

The enigma was resolved by us at the end of the same year using symmetry arguments and fundamental hydrodynamics (Cartwright et al., 2004). Because at the lateralization stage the embryo has already defined the dorsal-ventral and anterior-posterior asymmetry axes, the only chirally symmetric way to produce a chirally asymmetric flow is to use the tridimensional and pseudovectorial character of rotations (Fig. 1). This led us to conclude that the clockwise-rotating cilia should be tilted toward the posterior end of the node. By means of a simple minimal model of the system where the cilia were idealized as elementary point rotators termed rotlets, we were first able to rule out an existing alternative hypothesis that the origin of the flow was related to the shape of the node (Okada et al., 1999) and then to estimate the tilt angle of the cilia from the measured velocity of the flow and the observed rotation frequency of the cilia (Fig. 2). It is important to remark that this prediction predated the experimental confirmation that this tilt (Nonaka et al., 2005; Okada et al., 2005) indeed existed in real embryos and the actual measurement of the detailed structure of the cilia; even more notable was the quantitative confirmation of the tilt angle predicted a priori from our theory (Cartwright et al., 2004).

The history of this discovery is very instructive with respect to the possible ways that general physical thinking may best contribute to interdisciplinary endeavors in biology, such as the development of this subject. There is a strongly implanted tendency in applying physics to biology to build models a posteriori to describe mathematically the existing data. This modeling philosophy reduces the role of mathematical physics to a sort of painting tool to produce sophisticated pictures of a

\footnotetext{
Julyan Cartwright is from the Instituto Andaluz de Ciencias de la Tierra, CSIC-Universidad de Granada, Campus Fuentenueva, E-18071 Granada, Spain.

Nicolas Piro is from the ICFO-Institut de Ciencies Fotoniques, Mediterranean Technology Park, E-08860 Castelldefels (Barcelona), Spain.

Oreste Piro is from the Departmento de Fisica e Instituto de Fisica Interdisciplinar y Sistemas Complejos IFISC (CSIC-UIB), Universitat de les Illes Balears, E-07122 Palma de Mallorca, Spain.

Idan Tuval is from the Department of Applied Mathematics and Theoretical Physics, University of Cambridge, Cambridge CB3 OWA, UK.

*Correspondence to: Julyan H. E. Cartwright, Instituto Andaluz de Ciencias de la Tierra, CSIC-Universidad de Granada, Campus Fuentenueva, E-18071 Granada, Spain. E-mail: julyan@lec.csic.es, piro@ifisc.uib.es, I.Tuval@damtp.cam.ac.uk, nicolas.piro@icfo.es

Published online in Wiley InterScience (www.interscience.wiley.com). DOI: 10.1002/bdrc.20127
} 


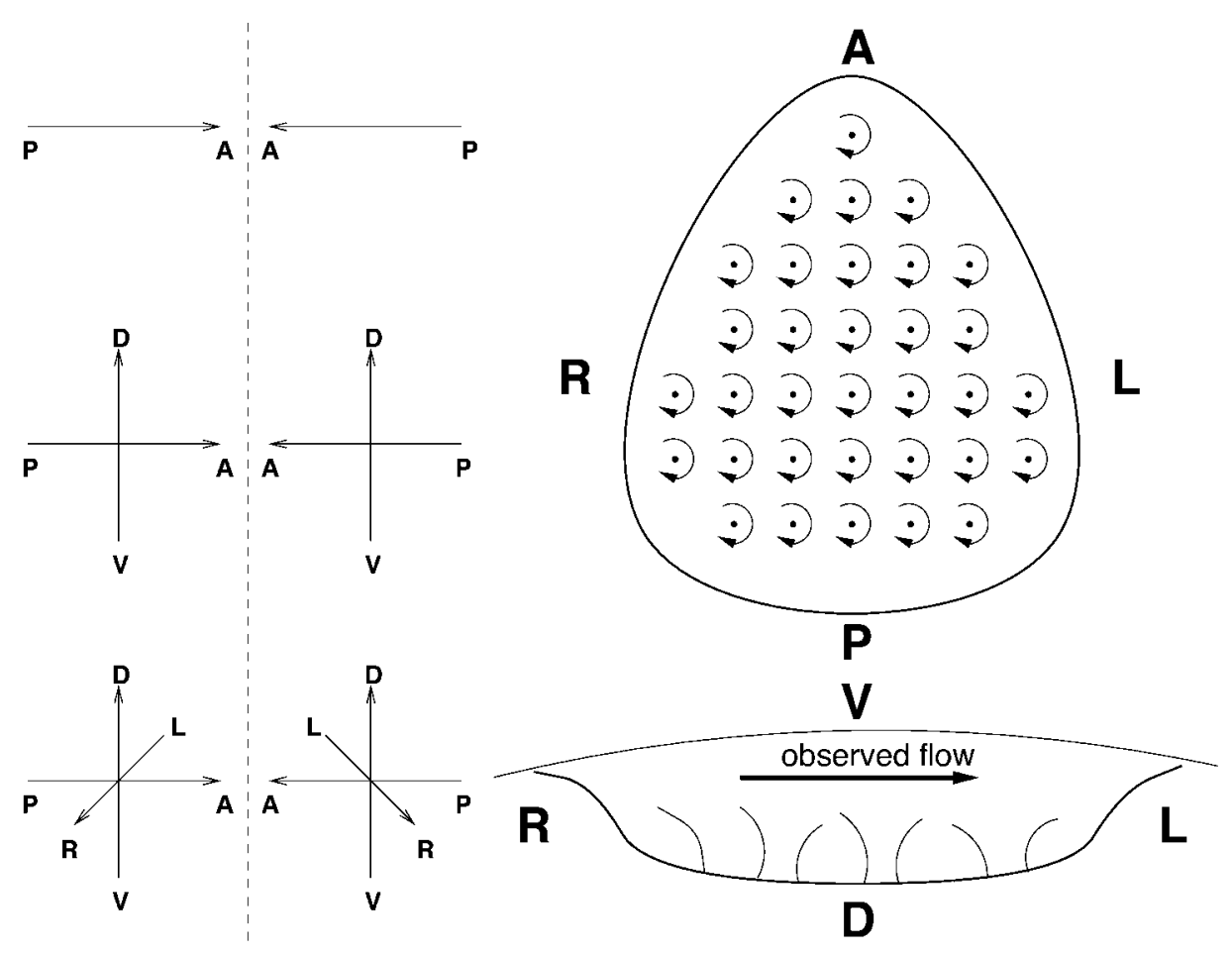

(a)

(b)

Figure 1. (a) Anterior-posterior, dorsal-ventral, and left-right axes provide a coordinate system for the vertebrate body plan. When only one or two of the axes are defined, the result is achiral-the mirror image is the same as the original. But when the final, left-right axis is added, there are now two chiral forms. (b) Ventral and posterior sketch views of the node of the mouse embryo, and its rotating monocilia, showing also the experimentally observed leftward nodal flow.

given phenomenon. But while a picture may be extremely beautiful, realistic, and detailed, it is just a picture, generally void of any predictive power to explore unknown aspects of the phenomenon. Our approach is essentially the opposite. In the same artistic metaphor, we advocate a more modernistic and abstract attitude, discarding useless details that distract the attention from the deep and fundamental aspects of a problem so that we can concentrate on well-defined creative proposals to look at a given problem in a new and different way. The prediction of a tilt angle somewhere between $25^{\circ}$ and $30^{\circ}$ by our simplified picture of the cilia-fluid interaction before the tilting was observed, and the experimental measurement of the angle in close agreement with our estimates is a very tangible proof of success of this approach.

One aspect of the system this work intentionally left aside was the analysis of the nearby boundary effects on the flow induced by tilted cilia within the node. When worrying about obtaining a welldefined estimate of the tilting angle in terms of the observed strength of the main flow, we need to focus on the sources of the flow and forget momentarily the complexity of the perturbations implied by the boundaries. The price to pay for such a radical simplification is, of course, the appearance of undesired symmetries that are broken by the boundaries. In our case, this implies the presence of a bogusly strong return flow accompanying the observed one. We, however, advanced that this bogus flow would be strongly suppressed once the boundary conditions were properly taken into account, as we later confirmed by performing full-scale fluid dynamical simulations of the whole system, taking into account not only boundary conditions but also the finite size of the rotating cilia (Cartwright et al., 2007).

The knowledge of the precise global structure of the nodal flow is still incomplete. For example, in spite of the impressive advance produced by the powerful experimental techniques that have recently been developed to directly visualize both the nodal flow and the cilia motion, these are deeply invasive in the functional sense, in mice at least. For instance, while a normally operational node is basically a closed chamber, in the experiments for visualizing the flow Reichert's membrane is removed; a maneuver that likely produces a radical modification of the flow from its in vivo conditions (Nonaka et al., 1998). This incomplete knowledge is a fundamental obstacle to achieve an unambiguous understanding of the mechanism by which the macroscopic structure of the flow produces a concentration gradient of the molecular agents 


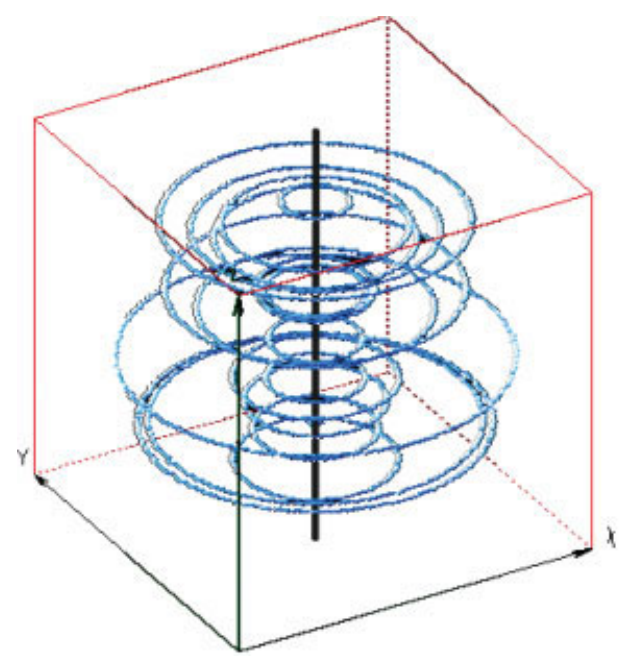

(a)

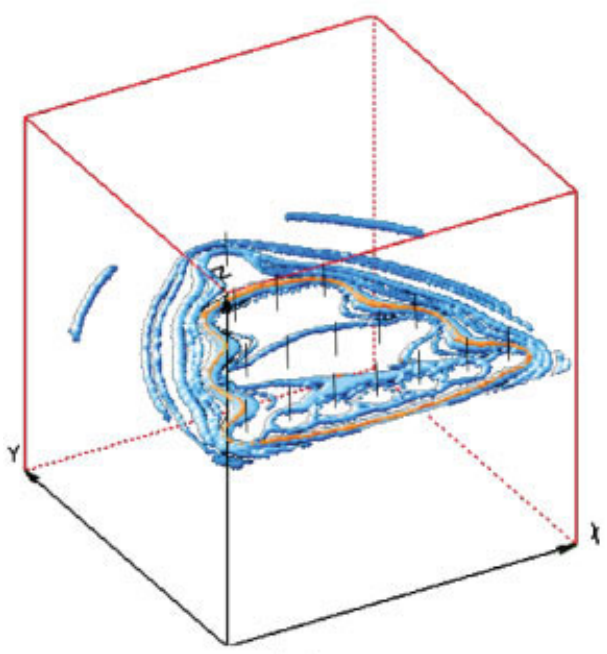

(c)

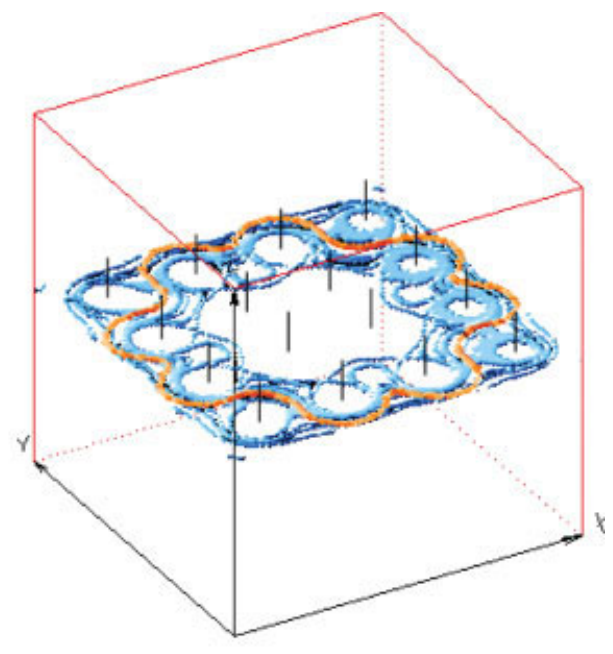

(b)

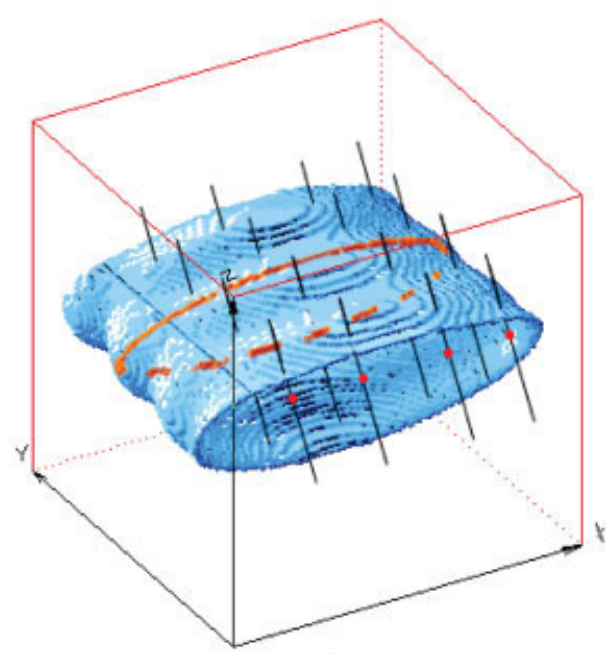

(d)

Figure 2. (a) Vortical flow structure produced by a single rotlet. (b) Rectangular array of rotlets with axes vertical, showing cellular structure of vortices with a general circulation only occurring at the edges. (c) Triangular array of rotlets with axes vertical, to correspond more closely with the shape of the node. As in (b), there is a general circulation only at the edges. (d) Result of tilting the rotlet axes: array of tilted rotlets with tilt angle $\alpha=24^{\circ}$, showing directional flow above and below the array.

responsible for the differentiation across the symmetry axis.

In the absence of such a precise characterization of the flow, but based on the generic behavior of Stokes flow in closed chambers, we suggested a plausible geometry that takes into account in a qualitative manner the presence of boundaries (Cartwright et al., 2004). This geometry, later confirmed by full-scale hydrodynamical simulations with realistic boundary conditions (Cartwright et al., 2007), consists of a strong and well-defined right-left flow induced by the cilia motion com- plemented by two much slower and chaotic return flow components required by mass conservation possibly located near the floor and the ceiling of the node. The latter component may be difficult to observe in experimental conditions where Reichert's membrane has been removed, while the former may be weak under physiological conditions and is also strongly conditioned by the stroking cilia, which makes it much more irregular. It is worth emphasizing that while the return flow is a trivial necessity imposed by mass conservation, the relative strength of the top and bottom flows depends on the length of the cilia compared to the depth of the node.

\section{TRANSMISSION OF FLOW INFORMATION}

This flow structure gives support to a possible robust mechanism of gradient formation for the concentration of a morphogen. Namely, the combination of three processes: advection or hydrodynamical transport of the morphogen, molecular-or another type of- 


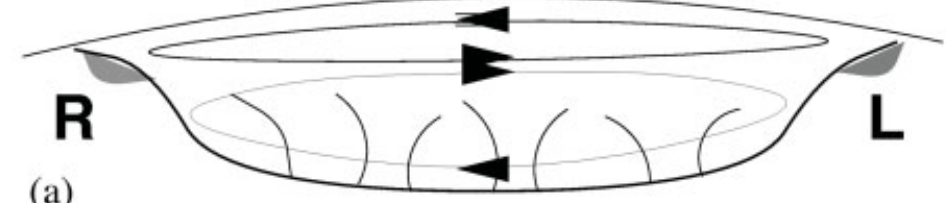

(a)
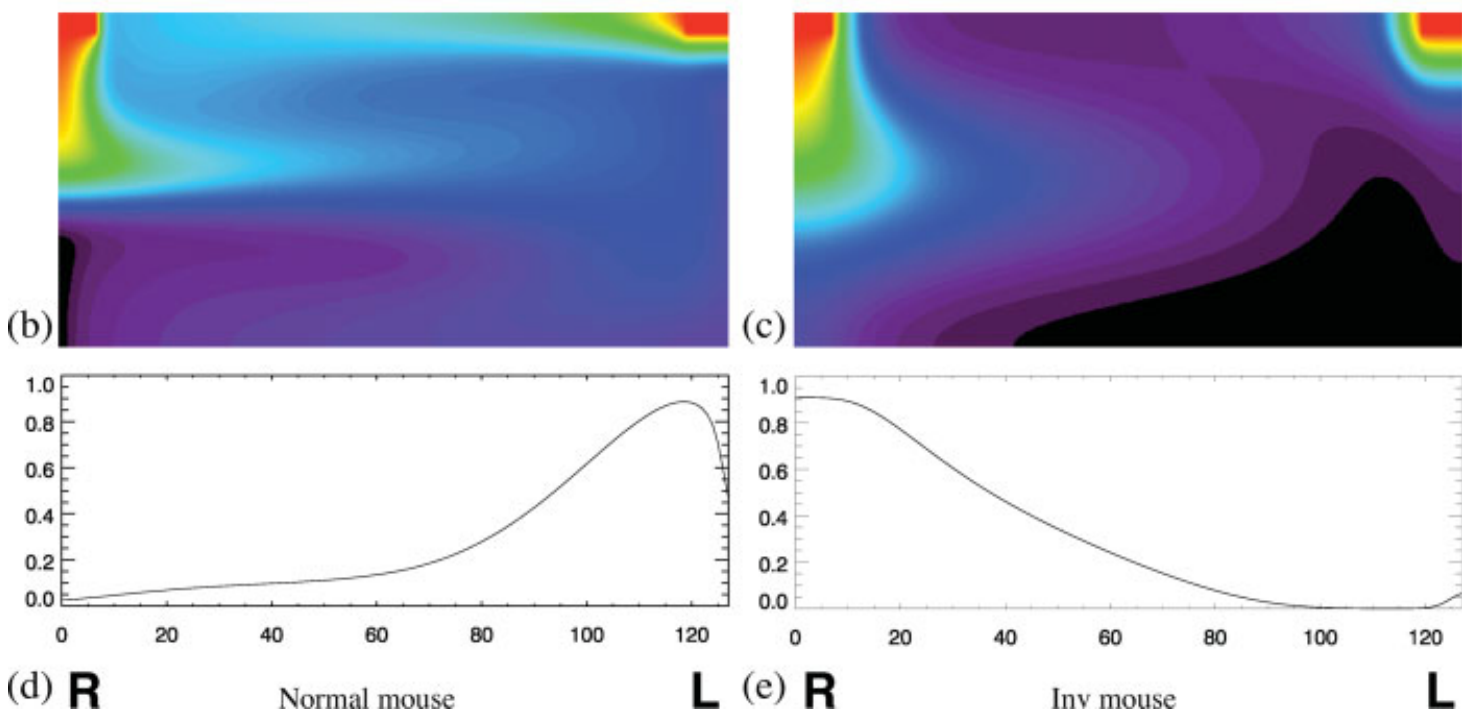

(d) $\mathbf{R}$

Normal mouse

$\mathbf{L}(\mathrm{e}) \mathbf{R}$

Inv mouse

Figure 3. (a) Sketch of how fluid will recirculate within the node in vivo with diffuse return flows above and below the more intense outward flow in the center. Also shown is the putative placement of morphogen sources (gray areas) at the left and right sides adjacent to the upper recirculatory vortex. Numerical simulations of our model depicting the steady-state concentration of a morphogen with a finite lifetime within the node with (b) normal and (c) Inv mice. The color scale is as for a rainbow, with red the highest concentration, and violet the lowest. Graphs of the concentration of morphogen at the floor of the node in the above simulations with (d) normal and (e) Inv mice (arbitrary units).

diffusion, and a chemical deactivation reaction. Mathematically, this is expressed in terms of a socalled reaction-advection-diffusion partial differential equation whose input should be the flow scheme including its characteristic intensity and the relevant diffusion and decomposition rates. If the balance between these three elements is correct, we have shown that a gradient in the morphogen concentration builds up in the appropriate direction to trigger the differentiation between the left and the right sides of the developing embryo (Cartwright et al., 2004) (Fig. 3). Moreover, alterations of this balance originating in the debilitation of the right-left flow outside of a given range are shown to produce the opposite gradient, which might explain the origin of the large proportions of situs inversus in some genetically originated situations, such as that of so-called Inv mice (Okada et al., 1999).
Even though this original proposal was built on the basic assumption that morphogens were secreted in a continuous way, which would imply that the diffusion rate to be included was indeed molecular diffusion, the model still holds with the recently discovered fact that the morphogen is released in a quantal fashion by microvilli, that is, in parcels wrapped by a lipid vesicle known as nodal vesicular parcels (NVPs) (Tanaka et al., 2005) (Fig. 4). The NVPs are naturally advected by the flow basically in the same way as individual fluid particles would be, but owing to their size, molecular diffusion has little effect on them. However, an effective diffusion rate stems from the disordered nature of the flow induced by the natural inhomogeneities of the node structure. On the other hand, the role of the deactivation reaction would be now played by an effective lifetime of the parcels. The trajectories of the NVPs can be computed supposing them to be perfect passive tracers released at random points above the floor of the node. This simulates their experimentally observed origin as vesicles projected into the flow by microvilli and released every 5 to 15 seconds (Tanaka et al., 2005). After the experimental observations, they are supposed to break when they collide with a wall or with a cilium. Statistics from our numerical model corresponding to some hours of nodal flow for the position within the node at the moment of rupture of a large number of NVPs are shown in histogram form in Figure 5. The outcome is similar for the in vivo and in vitro cases. Most of the NVPs are transported leftward across the node and collide with the left wall or with the cilia nearest to it. The smaller intermediate peaks in the histograms indicate that a few are broken in other locations across the floor of the node by interactions with cilia 


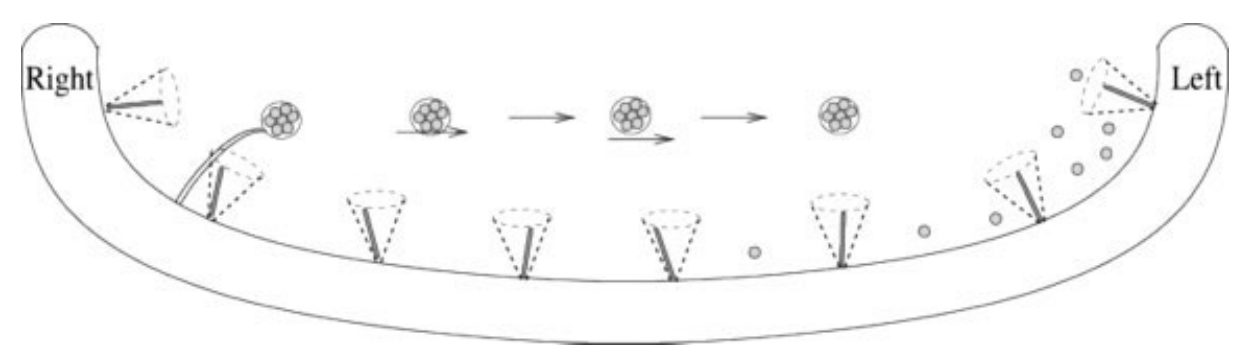

Figure 4. Sketch of a vertical slice across the node viewed from the ventral side showing the monocilia producing the leftward flow that transports nodal vesicular parcels. The mouse node is some $50 \mu \mathrm{m}$ across and from 10 to $20 \mu \mathrm{m}$ deep. Note that, following the convention in this field, in this and all other vertical slices of the node shown here the node is seen from the ventral side, and thus the left side of the embryo is on the viewer's right.
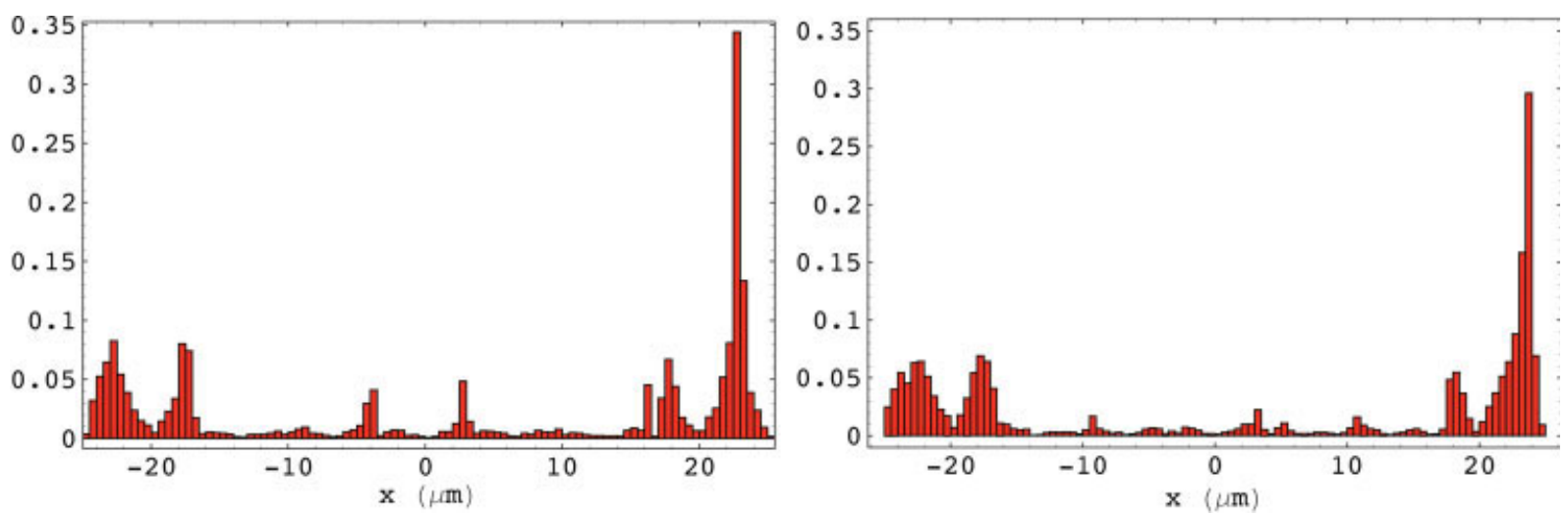

Figure 5. Histograms produced from our numerical model showing the relative frequency with which a nodal vesicular parcel breaks as a function of its position from right to left along the floor of the node. On the left is the in vivo case and on the right the in vitro flow.

there. The in vitro histogram shows a somewhat smaller main peak on the left side of the node than the in vivo case, although it is clear that in both instances the majority of NVPs break on the left side of the node. This means that no matter where the NVPs are released by the microvilli, and in particular if they are released in a symmetric fashion across the node, they will most probably break near the left wall and deliver their cargo of morphogen. Although this model is theoretically plausible, its validation or falsification requires a new generation of experiments designed to investigate the precise structure of the nodal flow in vivo, on one hand, and the fine details of the behavior of NVPs on the other. One can think, for example, of isolating nodal vesicular parcels to investigate their behavior and stability with respect to mechanical and biochemical forces under controlled conditions.

\section{DEVELOPMENTAL FLUID DYNAMICS}

Our interest in the problem of how left and right are determined in the vertebrate embryo stemmed on one hand from reading McManus' excellent book, "Left Hand, Right Hand" (McManus, 2002), and on the other hand from articles that appeared in the literature in 2002. In the 4th July issue of Nature that year, Nonaka et al. (2002) published a letter, "Determination of left-right patterning of the mouse embryo by artificial nodal flow." Their concluding sentences read "Of the questions that remain unanswered, one of the most important concerns the mechanism by which the vectorial flow is generated by the rotational movement of the cilia. Hydrodynamic considerations may provide some insight: it will be essential to characterize precisely the movement and morphology of the cilia, the shape of the node, and the hydrodynamic properties of the nodal flow." In the same issue, Stern (2002) commented on the Nonaka et al.'s letter in an article, "Fluid flow and broken symmetry," in which he noted that the nodal flow "highlights the significance of biomechanical phenomena in generating biological pattern." The research was also highlighted in Nature Science Update that week, in which Whitfield in a piece entitled "Embryos grow with the flow" noted that "Finding out how flow drives form is "the million-dollar question" (Whitfield, 2002). As physicists with a long interest and experience in biological problems, and with a back- 
ground in dynamical systems and in fluid dynamics, we decided to attack the problem. We had worked extensively with low Reynolds number fluid flow, as is present in the embryonic node, so we found ourselves ideally placed to investigate the physical aspects of the nodal-flow problem.

Biological symmetry breaking is a problem that has long interested physical scientists. In his pioneering work on the chemical basis of morphogenesis, Turing (1952) proposed the mechanism of pattern formation through the interaction of diffusing morphogens that now bears his name. He recognized the special problem for his theory posed by left-right symmetry breaking in vertebrates; how to explain that nature almost always breaks left-right symmetry in a given sense, whereas his mechanism would lead to approximately equal numbers of animals with situs inversus and situs solitus? He proposed that the input to the system must somehow be biased. Almirantis and Nicolis (1987) later showed in detail how an initial gradient could seed the process. Brown and Wolpert (1990) hypothesized a chiral molecule with a fixed orientation relative to the anterior-posterior and dorsal-ventral axes, which would provide the necessary information on left and right for the initial biasing. But nature, it seems, in mice at least, prefers to use not a chiral molecule, but a chiral structurea molecular motor-to provide advection in a given direction relative to the anterior-posterior and dorsal-ventral axes to seed the symmetry breaking. The information on which side is which is then carried to the rest of the embryo by diffusion. In fact, Hamada et al. (2002) proposed that the subsequent phase of propagation of the broken symmetry could function by exactly the mechanism Turing imagined, with the initial small concentration difference produced by the nodal flow magnified by a nonlinear interaction between two diffusing proteins, Nodal and Lefty. The interaction may involve the fluid flow itself, which would make this a biological instance of a recently proposed generalized Turing patternformation mechanism including fluid flow (Satnoianu et al., 2001).

We have discussed here experiments on mice, but similar structures to the node with its monocilia are found in other vertebrate embryos, so that it has been surmised that this left-right symmetry-breaking mechanism may be universal among vertebrates (Essner et al., 2002). On the other hand, experimental evidence has been put forward for asymmetries in chick and frog embryos before the emergence of the nodal structures (Mercola, 2003). If these observations prove to be correct, then nodal flow is not the earliest left-right symmetry breaking event in some vertebrates. If the initial symmetry-breaking mechanism differs across species, the chiral molecule or structure, equivalent to the chiral monocilium of the node, that bootstraps the process must be sought for those cases. Moreover, the role of the nodal flow in those species would need to be clarified: would it then be acting as a means to preserve or amplify an initial asymmetric signal from the earlier symmetry-breaking event, or would it constitute a second, independent, mechanism for determining left and right? Might this be a case of two independent mechanisms creating extra security through redundancy? More evidence needs to be collected.

Diffusion is ubiquitous in biology. Nature also often uses advection to achieve its ends, for example, in the cardiovascular system, and it has been recently found to be fundamental in the development of the heart (Hove et al., 2003). The use of cilia to move fluid is also common, for example, in the lung. Microbes use cilia and flagella for propulsion, just as the node uses them to advect fluid, and the similarity of scale implies a similarity of environment. This highlights the resemblance of the situation to that of microbial swimming. In both cases, we are talking of life at low Reynolds number (Purcell, 1977). The problems of moving fluids at the microscale, with their associated low Reynolds numbers, are also now interesting engineers who design fluid flow Microsystems, so-called microfluidics devices (Gad-el-Hak, 1999). We humans inhabit a world of much higher Reynolds number, and our intuition on how fluids behave is not straightforwardly transferable into this alien inertia less environment, which is why some ideas put forward for producing a directional flow from rotating cilia cannot work. In creeping flow, algorithms such as varying the angular velocity of the cilia in different parts of the rotation cycle do not obtain the desired effect, because there is no fluid inertia. Producing the nodal flow is not like waving your arms about in a swimming pool (Whitfield, 2002), but more akin to finding oneself "in a swimming pool that is full of molasses, and ... forbid[den] ... to move any part of [the] body faster than $1 \mathrm{~cm} / \mathrm{min}^{\prime \prime}$ (Purcell, 1977). In the nodal environment, nine orders of magnitude lower in Reynolds number than the above person in a swimming pool, the lack of inertia constrains the fluid physics that the biology can exploit, leaving our proposed mechanisms of a posterior tilt of the cilia and the chemosensing of the flow as the best hypotheses, compatible with the facts, for producing the observations reported in experiments.

\section{QUO VADIMUS?}

The direction of rotation of $a$ monocilium is determined by the motor proteins that power its molecular motor. These, like the vast majority of naturally occurring proteins, are made up of chiral amino acids in just one of their two possible forms: they are all laevo. An equivalent molecular motor using the same amino acids in their opposite, dextro, configuration would rotate in the reverse direction. In this way, left-right symmetry breaking here is ultimately determined by the chirality of natural amino acids, not directly through a chiral molecule, but by setting the direction of the nodal 
flow. There are several schools of thought as to how this natural chirality has arisen. One supposes it to be a frozen accident, and that life could equally have chosen to use dextro amino acids, but a competing idea points out that laevo amino acids are slightly more stable than the dextro forms, owing to the broken parity of the weak nuclear force (Mason, 1984). This provides us with the fascinating idea that we may have our hearts on the left because, as Wolfgang Pauli famously put it, God is weakly left handed (McManus, 2002). But, whether or not there turns out to be a causal link between parity violation and the asymmetry of the vertebrate body plan, after answering the how part of the symmetry-breaking problem, the most intriguing question that remains may be: why does nature take care to break the symmetry in a given direction, rather than leaving things to chance and allowing half the population to have situs inversus? Why is it, as Aristotle observed over two millennia ago, preternatural for left-right patterning to be transposed?: "on the right-hand side lies the 'liver', and on the left-hand side the 'spleen', alike in all animals that are provided with these organs in an ordinary and not preternatural way; for, be it observed, in some quadrupeds these organs have been found in a transposed position" (Aristotle $350 \mathrm{BC}$ ).

\section{REFERENCES}

Almirantis Y, Nicolis G. 1987. Morphogenesis in an asymmetric medium. Bull Math Biol 49:519-538.

Aristotle, 350BC. The History of Animals. Translated by D'Arcy Wentworth Thompson.

Brown NA, Wolpert L. 1990. The development of handedness in left/right asymmetry. Development 109:1-9.

Cartwright JHE, Piro O, Tuval I. 2004. Fluid-dynamical basis of the embryonic development of left-right asymmetry in vertebrates. Proc Natl Acad Sci 101:7234-7239.

Cartwright JHE, Piro N, Piro O, Tuval I. 2007. Embryonic nodal flow and the dynamics of nodal vesicular parcels. J R Soc Interf 4:49-55.

Essner JJ, Vogan KJ, Wagner MK, et al. 2002. Left-right development: conserved function for embryonic nodal cilia. Nature 418:37-38.

Gad-el-Hak M. 1999. The fluid mechanics of microdevices. J Fluids Eng 121:5-33.

Hamada $\mathrm{H}$, Meno C, Watanabe D, Saijoh Y. 2002. Establishment of vertebrate left-right asymmetry. Nat Rev Genet 3:103-113.

Hove JR, Köster RW, Forouhar AS, et al. 2003. Intracardiac fluid forces are an essential epigenetic factor for embryonic cardiogenesis. Nature 421 : 172-177.

Mason SF. 1984. Origins of biomolecular handedness. Nature 311:19-23.

McManus C. 2002. Right hand, left hand. Weidenfeld \& Nicholson, London.

Mercola M. 2003. Left-right asymmetry: nodal points. J Cell Sci 116: 3251-3257.
Nonaka S, Shiratori H, Saijoh Y, Hirokawa N. 2002. Determination of leftright patterning of the mouse embryo by artificial nodal flow. Nature 418:96-99.

Nonaka S, Tanaka Y, Okada Y, et al. 1998. Randomization of left-right asymmetry due to loss of nodal cilia generating leftward flow of extraembryonic fluid in mice lacking KIF3B motor protein. Cell 95:829-837.

Nonaka S, Yoshiba S, Watanabe D, et al. 2005. De novo formation of leftright asymmetry by posterior tilt of nodal cilia. Plos Biol 3:e268.

Okada Y, Nonaka S, Tanaka $Y$, et al. 1999. Abnormal nodal flow precedes situs inversus in iv and inv mice. Mol Cell 4:459-468.

Okada Y, Takeda S, Tanaka Y, et al. 2005. Mechanism of nodal flow: a conserved symmetry breaking event in left-right axis determination. Cell 121:633-644.

Purcell EM. 1977. Life at low Reynolds number. Am J Phys 45:3-11.

Satnoianu RA, Maini PK, Menzinger M. 2001. Parameter space analysis, pattern sensitivity and model comparison for Turing and stationary flow-distributed waves (FDS). Phys D 160:79-102.

Stern CD. 2002. Fluid flow and broken symmetry. Nature, 418:29-30.

Tanaka Y, Okada Y, Hirokawa N. 2005. FGF-induced vesicular release of Sonic hedgehog and retinoic acid in leftward nodal flow is critical for left-right determination. Nature 435: 172-177.

Turing AM. 1952. The chemical basis of morphogenesis. Phil Trans $R$ Soc Lond B 237:37-72.

Whitfield J. 2002. Embryos grow with the flow. Nat Sci Update July 4, 2002 [doi: 10.103/news02701-7]. 